archives-ouvertes

\title{
Generalized Solutions of Hamilton - Jacobi Equation to a Molecular Genetic Model
}

\author{
Nina Subbotina, Lyubov Shagalova
}

\section{To cite this version:}

Nina Subbotina, Lyubov Shagalova. Generalized Solutions of Hamilton - Jacobi Equation to a Molecular Genetic Model. 27th IFIP Conference on System Modeling and Optimization (CSMO), Jun 2015, Sophia Antipolis, France. pp.462-471, 10.1007/978-3-319-55795-3_44 . hal-01626917

\section{HAL Id: hal-01626917 https://hal.inria.fr/hal-01626917}

Submitted on 31 Oct 2017

HAL is a multi-disciplinary open access archive for the deposit and dissemination of scientific research documents, whether they are published or not. The documents may come from teaching and research institutions in France or abroad, or from public or private research centers.
L'archive ouverte pluridisciplinaire HAL, est destinée au dépôt et à la diffusion de documents scientifiques de niveau recherche, publiés ou non, émanant des établissements d'enseignement et de recherche français ou étrangers, des laboratoires publics ou privés. 


\title{
Generalized Solutions of Hamilton - Jacobi Equation to a Molecular Genetic Model
}

\author{
Nina Subbotina ${ }^{1,2}$ and Lyubov Shagalova ${ }^{1}$ \\ 1 Krasovskii Institute of Mathematics and Mechanics, Ural Branch of RAS \\ S. Kovalevskaya str., 16, 620990 Ekaterinburg, Russia \\ subb@uran.ru, shag@imm.uran.ru \\ 2 Ural Federal University named after the first President \\ of Russia B.N. Yeltsin, Ekaterinburg, Russia
}

\begin{abstract}
A boundary value problem with state constraints is under consideration for a nonlinear noncoercive Hamilton-Jacobi equation. The problem arises in molecular biology for the Crow - Kimura model of genetic evolution. A new notion of continuous generalized solution to the problem is suggested. Connections with viscosity and minimax generalized solutions are discussed. In this paper the problem is studied for the case of additional requirements to structure of solutions. Constructions of the solutions with prescribed properties are provided and justified via dynamic programming and calculus of variations. Results of simulations are exposed.
\end{abstract}

Keywords: Hamilton - Jacobi equation, method of characteristics, generalized solutions, viscosity solutions, state constraints

\section{Introduction}

In [1] a new way to study molecular evolution has been proposed. According to this way dynamics of the Crow - Kimura model for molecular evolution can be analyzed via the following HJE

$$
\partial u / \partial t+H(x, \partial u / \partial x)=0,
$$

where the Hamiltonian $H(\cdot)$ has the form

$$
H(x, p)=-f(x)+1-\frac{1+x}{2} e^{2 p}-\frac{1-x}{2} e^{-2 p} .
$$

The function $f(\cdot)$ in $(2)$ is given and called fitness. Equation (1) is considered for $t \geq 0,-1 \leq x \leq 1$. It is also assumed that an initial function $u_{0}: \mathbb{R} \rightarrow \mathbb{R}$ is given such that

$$
u(0, x)=u_{0}(x), \quad x \in[-1 ; 1] .
$$

In [1] problem (1)-(3) was studied for input data $u_{0}(x)=-a\left(x-x_{0}\right)^{2}, a>0$, $f(x)=x^{2}$ and physical interpretations were used. 
The classical method for solving PDE of the first order in Cauchy problem is the method of characteristics (see, e.g. [2]). This method reduces integration of PDEs to integration of the characteristic system of ODEs.

The characteristic system for problem (1)-(3) has the form

$$
\begin{gathered}
\dot{x}=H_{p}(x, p)=-(1+x) e^{2 p}+(1-x) e^{-2 p}, \\
\dot{p}=-H_{x}(x, p)=f^{\prime}(x)+\left(e^{2 p}-e^{-2 p}\right) / 2, \\
\dot{z}=p H_{p}(x, p)-H(x, p),
\end{gathered}
$$

with initial conditions

$$
x(0, y)=y, \quad p(0, y)=u_{0}^{\prime}(y), \quad z(0, y)=u_{0}(y), \quad y \in[-1 ; 1] .
$$

Here $H_{x}(x, p)=\partial H(x, p) / \partial x, \quad H_{p}(x, p)=\partial H(x, p) / \partial p, f^{\prime}(x)=\partial f(x) / \partial x$. Solutions of the system (4)-(5) are called characteristics. Components $x(\cdot, y)$, $p(\cdot, y)$ and $z(\cdot, y)$ of the solution are called state, conjugate, and value characteristics, respectively.

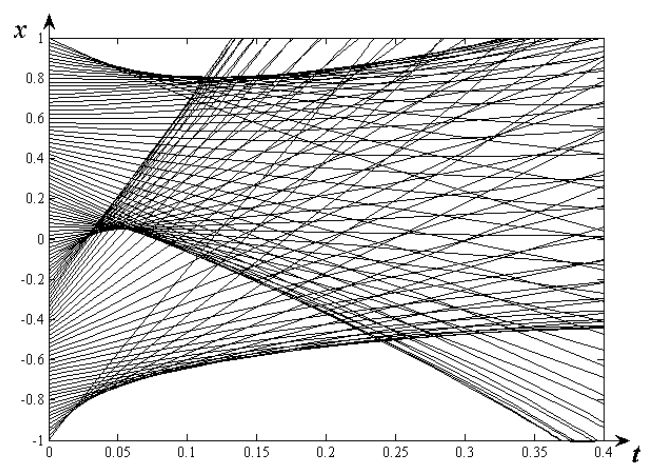

Fig. 1. State characteristics for the case $f(x)=-0.25 x^{2}, u_{0}(x)=0.25(x-0.5)^{2}-$ $0.1 \cos 2 \pi x$.

The method of characteristics can be applied to constructions of solutions for problem (1)-(3) in such a neighborhood of the initial manifold (5) where state characteristics don't cross. As a rule, characteristics for problem (1)-(3) are nonextendable to the whole time axis and can cross each other. Moreover, there are points in strip $t \geq 0,-1 \leq x \leq 1$. where solution of (1)-(3) should be found, and where the state characteristics do not pass. An example of such a behavior of state characteristics is presented on Fig. 1.

So, one can see that solutions of the problem (1)-(3) should be understood in a generalized sense. 
In [3], we introduced a concept of continuous generalized solutions (see Definition 1 below) and proved it's existence in problem (1)-(3) using tools of Nonsmooth Analysis and results of the Optimal Control Theory. It was also shown that the generalized solution is not unique.

In this paper, we consider problem (1)-(3) with additional requirements to the structure of solutions, see [4] and [5]. Namely, we need to construct a continuous solution in the strip $t \geq 0,-1 \leq x \leq 1$ in such a way that it coincides with a solution obtained by the method of characteristics in a domain part where the characteristics defined by (4) and (5) pass.

The paper is organized as follows. In Section 2, the definition of a continuous generalized solution is introduced, and the results on its existence are presented. In Section 3 we state the problem of constructing the generalized solution with prescribed properties, give sufficient conditions under which the problem can be solved, and formulate auxiliary results on which solving is based. A scheme for constructing the generalized solution and results of a simulation are presented in Section 4 and Section 5 respectively. And, in Section 6, we compare our generalized solution with viscosity solutions.

\section{Continuous Generalized Solutions to the Problem with State Constraints}

\subsection{The Problem with State Constraints and Definition of a Generalized Solution}

Let $T>0$ be such an instant that characteristics (4), (5) are extendable up to $T$, and $x(\cdot, y), p(\cdot, y), z(\cdot, y)$ are continuous on $[0, T]$ for all $y \in[-1 ; 1]$. Exact estimates for intervals of extendibility are obtained in $[4,6]$.

We consider problem (1)-(3) on the restricted closed domain

$$
\bar{\Pi}_{T}=[0 ; T] \times[-1 ; 1],
$$

and also use the notations

$$
\Pi_{T}=(0 ; T) \times(-1 ; 1), \quad \Gamma_{T}=\{(t, x) \mid t \in(0, T), x= \pm 1\} .
$$

In the HJEs' theory various concepts of generalized solutions have been introduced (see, e.g. [7-9]). Note that definitions of generalized solutions to HJEs in open areas were applied to problems with state constraints as additional requirements to solutions on the border were imposed. These requirements play a role of boundary conditions. Unfortunately, results of the theories of generalized solutions are inapplicable to the problem (1)-(3). In particular, one of the key conditions under which the known theorems on existense of a generalized viscosity solution $[8,10]$ has been proved is the coercivity of the Hamiltonian (see (22) below). And the theory of minimax solutions [9] is not developed for problems with state constraints. So, below a new definition of a generalized solution is introduced [3]. This definition is based on the minimax and viscosity approahes and uses the following tools of nonsmooth analysis $[10,11]$. 
Let $W$ be a set in $\mathbb{R}^{2}$. Denote by $\bar{W}$ the closure of this set, by $C(W)$ - the class of functions continuous on the set $W$.

Let $u(\cdot) \in C(\bar{W})$ and $(t, x) \in \bar{W}$. The subdifferential of the function $u(\cdot)$ at $(t, x)$ is the set

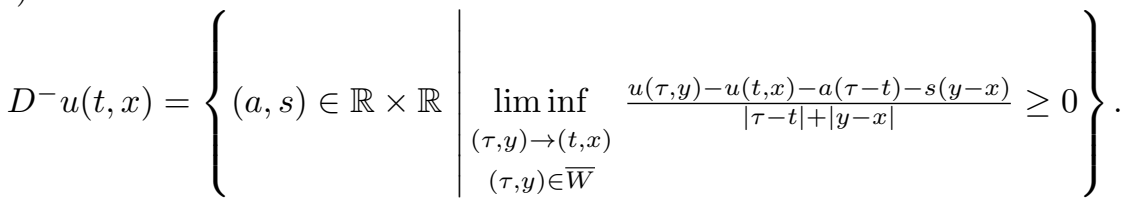

The superdifferential of the function $u(\cdot)$ at $(t, x)$ is the set

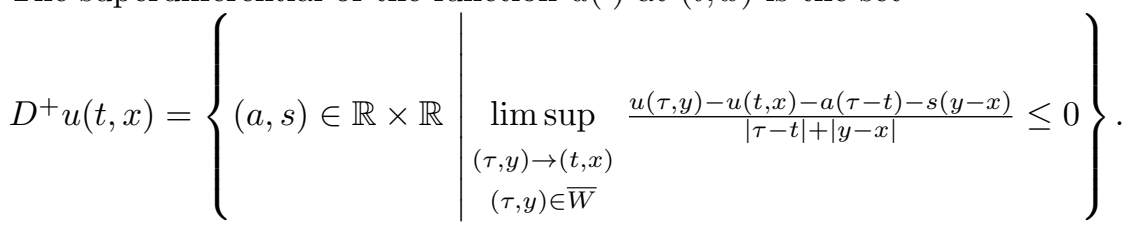

Let $\operatorname{Dif}(u)$ be the set of points where the function $u(\cdot) \in C(\bar{W})$ is differentiable. For a given set $M \subset \mathbb{R}^{2}$, the symbol co $M$ means its convex hull [13]. Let us define the set

$$
\begin{aligned}
\partial u(t, x)=\operatorname{co}\left\{(a, s) \mid a=\lim _{i \rightarrow \infty} \frac{\partial u\left(t_{i}, x_{i}\right)}{\partial t}, s=\lim _{i \rightarrow \infty} \frac{\partial u\left(t_{i}, x_{i}\right)}{\partial x} ;\right. \\
\left.\left(t_{i}, x_{i}\right) \rightarrow(t, x) \text { as } i \rightarrow \infty, \quad\left(t_{i}, x_{i}\right) \in \operatorname{Dif}(u)\right\} .
\end{aligned}
$$

Definition 1. A continuous function $u(\cdot): \bar{\Pi}_{T} \rightarrow \mathbb{R}^{2}$ is called a generalized solution to problem (1)-(3) iff it satisfies the initial condition (3) and the following relations are true

$$
\begin{gathered}
a+H(x, s) \leq 0, \quad \forall(a, s) \in D^{+} u(t, x), \forall(t, x) \in \Pi_{T}, \\
a+H(x, s) \geq 0, \quad \forall(a, s) \in D^{-} u(t, x), \forall(t, x) \in \Pi_{T}, \\
a+H(x, s) \geq 0, \quad \forall(a, s) \in D^{-} u(t, x) \cap \partial u(t, x), \forall(t, x) \in \Gamma_{T} .
\end{gathered}
$$

\subsection{Existence of Generalized Solutions}

The following statement was proved in [4] by using tools of Mathematical Theory of Optimal Control [14] and the method of generalized characteristics $[15,16]$.

Theorem 1. Let input data $u_{0}(\cdot):[-1,1] \rightarrow \mathbb{R}$ and $f(\cdot):[-1,1] \rightarrow \mathbb{R}$ be continuously differentiable functions. Let a function $\varphi(t, x): \mathbb{R}^{2} \rightarrow \mathbb{R}$ be also continuously differentiable and satisfy the relations

$$
\begin{gathered}
\varphi(0, x)=u_{0}(x) \quad \forall x \in[-1,1] ; \\
\frac{\partial \varphi(t, \pm 1)}{\partial t}+H\left( \pm 1, \frac{\partial \varphi(t, \pm 1)}{\partial x}\right)=0 \quad \forall t \geq 0 .
\end{gathered}
$$

Then there exists a solution $u(t, x)$ of problem (1)-(3) in sense of definition 1. The solution has the form

$$
u(t, x)=\max _{x\left(t, y^{\sharp}\right)=x}\left[\varphi\left(t^{\sharp}, y^{\sharp}\right)+\right.
$$




$$
\left.+\int_{t^{\sharp}}^{t} p\left(\tau, y^{\sharp}\right) H_{p}\left(x\left(\tau, y^{\sharp}\right), p\left(\tau, y^{\sharp}\right)\right)-H\left(x\left(\tau, y^{\sharp}\right), p\left(\tau, y^{\sharp}\right)\right) d \tau\right],
$$

for all $(t, x) \in \bar{\Pi}_{T}$, where $t^{\sharp} \in[0, T]$. If $t^{\sharp}=0$ then $y^{\sharp}=y \in[-1,1]$; if $t^{\sharp}>0$, then $y^{\sharp}= \pm 1$. The functions $\left(x\left(\cdot, y^{\sharp}\right), p\left(\cdot, y^{\sharp}\right)\right):\left[t^{\sharp}, t\right] \rightarrow \mathbb{R}^{2}$ are solutions for the system composed of the first two equations of characteristic system (4) with initial conditions

$$
x\left(t^{\sharp}, y^{\sharp}\right)=y^{\sharp}, \quad p\left(t^{\sharp}, y^{\sharp}\right)=\frac{\partial \varphi\left(t^{\sharp}, y^{\sharp}\right)}{\partial y}=p_{0}\left(t^{\sharp}, y^{\sharp}\right) .
$$

To obtain $u(t, x)$ in accordance with (10), one should consider the set of all state characteristics $x\left(\cdot, y^{\sharp}\right)$ passing through the point $(t, x)$, namely, $x\left(t, y^{\sharp}\right)=$ $x$. Note that the generalized solution to problem (1)-(3) is not unique because of wide choice of functions $\varphi(\cdot)$ in Theorem 1 .

\section{Solutions with Prescribed Properties}

Here, we consider a problem to construct the generalized solution of some particular structure.

Let $x^{-}(t)=x(t,-1)$ and $x^{+}(t)=x(t,+1), t \in[0, T]$ be the state characteristics started at $t=0$ from the points $x=-1$ and $x=1$, respectively. Below, we assume that the following condition is satisfied.

A. For the state characteristics $x(\cdot, y)$ with initial conditions $(5)$ at $t=0$ the inequalities are valid

$$
-1 \leq x^{-}(t) \leq x(t, y) \leq x^{+}(t) \leq 1, \quad \forall y \in[-1,1], \forall t \in[0, T] .
$$

Define the subdomains

$$
\begin{gathered}
G_{0}=\left\{(t, x) \mid t \in[0, T], x^{-}(t) \leq x \leq x^{+}(t)\right\} . \\
G_{+}=\left\{(t, x) \mid t \in[0, T], x^{+}(t) \leq x \leq 1\right\}, \\
G_{-}=\left\{(t, x) \mid t \in[0, T],-1 \leq x \leq x^{-}(t)\right\} .
\end{gathered}
$$

So, under the assumption $\mathbf{A}$, we get

$$
\bar{\Pi}_{T}=G_{+} \cup G_{0} \cup G_{-} .
$$

The goal of the work is to construct the generalized solution to problem (1)(3) such that it has the following form in $G_{0}$ :

$$
u(t, x)=\max _{x(t, y)=x}\left[u_{0}(y)+\int_{0}^{t} p(\tau) H_{p}(x(\tau), p(\tau))-H(x(\tau), p(\tau)) d \tau\right],
$$

where $x(t)=x(t, y), \quad p(t)=p(t, y), t \geq 0$, are state and conjugate characteristics, respectively, which satisfy at $t=0$ the initial conditions

$$
x(0, y)=y, \quad p(0, y)=u_{0}^{\prime}(y), \quad y \in[-1,1] .
$$




\subsection{Sufficient Conditions}

To solve the problem (1)-(3) with the requirement (12) we introduce the following additional assumptions on input data.

B1. The derivative $u_{0}^{\prime}(\cdot):[-1,1] \rightarrow \mathbb{R}$ is continuous and satisfies the inequalities

$$
u_{0}^{\prime}(1)<0, \quad u_{0}^{\prime}(-1)>0 .
$$

B2. The derivative $f^{\prime}(\cdot):[-1,1] \rightarrow \mathbb{R}$ is continuous and monotone nondecreasing. It satisfies the inequalities

$$
2 f^{\prime}(1)+e^{2 u_{0}^{\prime}(1)}<e^{-2 u_{0}^{\prime}(1)}, \quad-2 f^{\prime}(-1)+e^{-2 u_{0}^{\prime}(-1)}<e^{2 u_{0}^{\prime}(-1)} .
$$

\subsection{Auxiliary Problems of Calculus of Variations}

Consider the following two problems of Calculus of Variations over the set of all continuously differentiable functions $x(\cdot):[0, T] \rightarrow \mathbb{R}$

$$
\begin{aligned}
& I(x(\cdot))=\int_{0}^{\bar{t}} H^{*}(x(\tau), \dot{x}(\tau)) d \tau \mapsto \max \\
& x_{1}(0)=1, \quad x_{1}(\bar{t})=\bar{x}, \quad(\bar{t}, \bar{x}) \in G_{+} ; \\
& x_{2}(0)=-1, \quad x_{2}(\bar{t})=\bar{x}, \quad(\bar{t}, \bar{x}) \in G_{-}
\end{aligned}
$$

where

$$
H^{*}(x(t), \dot{x}(t))=\inf _{p \in R}[p \dot{x}(t)-H(x(t), p)] .
$$

The following assertions are proven in $[4,5]$, where conditions B1-B2 are essential.

Theorem 2. For any interior point $(\bar{t}, \bar{x}) \in G_{+}$there exists a unique extremal $x=x(t)$ of the problem (13),(14),(16). The extremal coinsides with a state characteristic $x\left(\cdot ; 0,1, p_{0}\right)$ satisfying the initial conditions $x(0)=1, \quad p(0)=p_{0} \in$ $\left(-\infty, u_{0}^{\prime}(1)\right)$ where initial value $p_{0}$ can be defined uniquely from the condition $x(\bar{t})=\bar{x}$. The functional (13) attains its strong maximum at this extremal.

Theorem 3. For any interior point $(\bar{t}, \bar{x}) \in G_{-}$there exists a unique extremal $x=x(t)$ of the problem (13),(15),(16). The extremal coinsides with a state characteristic $x\left(\cdot ; 0,-1, p_{0}\right)$ satisfying the initial conditions $x(0)=-1, \quad p(0)=p_{0} \in$ $\left(u_{0}^{\prime}(-1), \infty\right)$ where initial value $p_{0}$ can be defined uniquely from the condition $x(\bar{t})=\bar{x}$. The functional (13) attains its strong maximum at this extremal.

Theorem 4. For any boundary point $(\bar{t}, 1), 0<\bar{t} \leq T$ in $G_{+}$, the maximum of functional (13), (16) is attained at a state characteristic $x\left(\cdot ; 0,1, p_{0}\right)$, such that the characteristics $x\left(\cdot ; 0,1, p_{0}\right), p\left(\cdot ; 0,1, p_{0}\right)$ are nonextendable from the interval $\left[0, t^{*}\left(p_{0}\right)=\bar{t}\right)$ to the right. The maximum of functional (13), (16) is equal to $I\left(x\left(\cdot ; 0,1, p_{0}\right)\right)=(f(1)-1) \bar{t}$. 
Theorem 5. For any boundary point $(\bar{t},-1), 0<\bar{t} \leq T$ in $G_{-}$, the maximum of the functional (13)-(16) is attained at a state characteristic $x\left(\cdot ; 0,-1, p_{0}\right)$, such that characteristics $x\left(\cdot ; 0,-1, p_{0}\right), p\left(\cdot ; 0,-1, p_{0}\right)$ are nonextendable from the interval $\left[0, t^{*}\left(p_{0}\right)=\bar{t}\right)$ to the right. The maximum of functional $(13),(16)$ is equal to $I\left(x\left(\cdot ; 0,-1, p_{0}\right)\right)=(f(-1)-1) \bar{t}$.

\section{Construction of the generalized solution}

The generalized solution of the problem (1)-(3) with the prescribed property (12) has the following form in $G_{0}$ :

$$
u(t, x)=\max _{x(t, y)=x}\left[\int_{0}^{t} p(\tau) H_{p}(x(\tau), p(\tau))-H(x(\tau), p(\tau)) d \tau+u_{0}(y)\right],
$$

where $x(t)=x(t, y), \quad p(t)=p(t, y), t \geq 0$, are state and conjugate characteristics satisfied initial conditions

$$
x(0, y)=y, \quad p(0, y)=\partial u_{0}(y) / \partial x, \quad y \in[-1,1] .
$$

Let $\left(t_{*}, x_{*}\right) \in G_{+}$and $x_{*}<1$. We assign

$$
u\left(t_{*}, x_{*}\right)=u_{0}(1)+\int_{0}^{t_{*}}\left[p(\tau) H_{p}(x(\tau), p(\tau))-H(x(\tau), p(\tau))\right] d \tau,
$$

where $x(t)=x^{+}\left(t, p_{0}\left(t_{*}, x_{*}\right)\right), p(t)=p^{+}\left(t, p_{0}\left(t_{*}, x_{*}\right)\right)$ is the solution of the problem of Calculus Variations (13),(14),(16).

For $x_{*}=1,0 \leq t_{*} \leq T$, we set

$$
u\left(t_{*}, 1\right)=u_{0}(1)+(f(1)-1) t_{*} .
$$

Let $\left(t_{*}, x_{*}\right) \in G_{-}$and $x_{*}>-1$. We assign

$$
u\left(t_{*}, x_{*}\right)=u_{0}(-1)+\int_{0}^{t_{*}}\left[p(\tau) H_{p}(x(\tau), p(\tau))-H(x(\tau), p(\tau))\right] d \tau,
$$

where $x(t)=x^{-}\left(t, p_{0}\left(t_{*}, x_{*}\right)\right), p(t)=p^{-}\left(t, p_{0}\left(t_{*}, x_{*}\right)\right)$ is the solution of the problem of Calculus Variations (13),(15),(16).

For $x_{*}=-1,0 \leq t_{*} \leq T$, we set

$$
u\left(t_{*},-1\right)=u_{0}(-1)+(f(-1)-1) t_{*} .
$$

So, we have defined function $u(\cdot)$ for all points from $\bar{\Pi}_{T}$ by relations (17)(21). Following the Cauchy method of characteristics [2], one can show that $u(\cdot)$ is continuously differentiable at any interior point $(t, x) \in G_{+} \cup G_{-}$, and the 
gradient of $u(\cdot)$ is equal to $(-H(x, p(t)), p(t))$. Theorems $2-5$ imply that $u(\cdot)$ is continuous in $\bar{\Pi}_{T}$, and inequalities (6)-(7) are valid. Below, in section 6.2 , we will show that $D^{-} u(t, x) \cap \partial u(t, x)=\varnothing, \quad(t, x) \in \Gamma_{T}$. So, $u(\cdot)$ is a generalized solution of problem (1)-(3) in sense of Definition 1. It follows from (17) that $u(\cdot)$ satisfies the prescribed property (12).

\section{$5 \quad$ Numerical example}

Results of simulation for the input data $u_{0}(x)=-0.02 x^{2}+0.001 \cos 2 \pi x, f(\cdot)=$ $-0.5 x^{2}$ are presented in Fig. 2. One can easily check that these input data satisfy the conditions B1, B2.
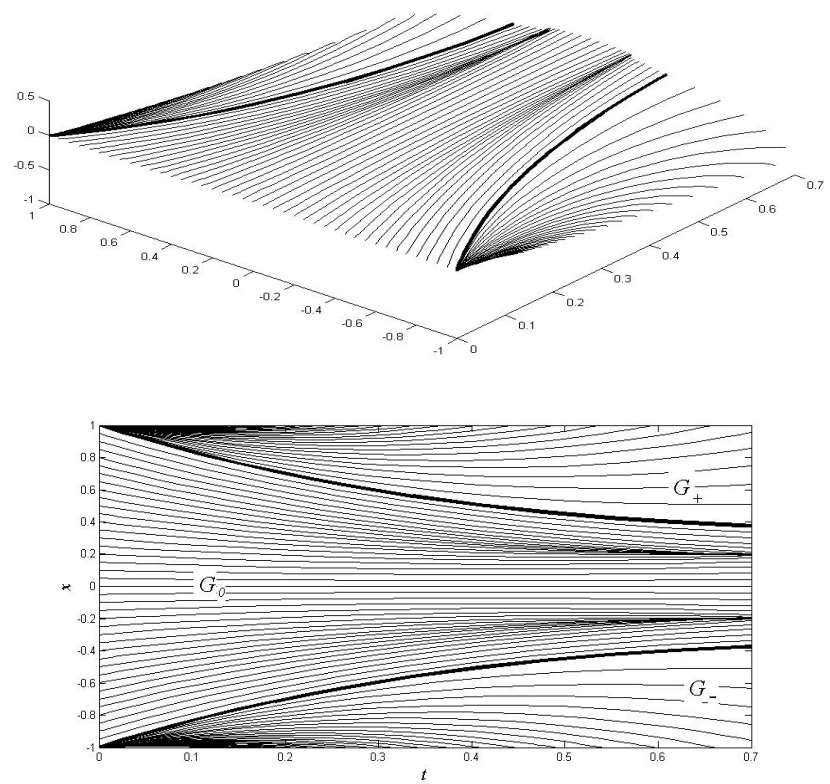

Fig. 2. The graph of the generalized solution and its projection on the $(t, x)$-plane for the input data $u_{0}(x)=-0.02 x^{2}+0.001 \cos 2 \pi x, f(x)=-0.5 x^{2}$.

\section{Comparison with Viscosity Solution}

One can see that Definition 1 coincides with the definition of viscosity solution in the interior points of the region $\bar{\Pi}_{T}$. The difference between these definitions is evident at boundary points, namely, on the set $\Gamma_{T}$. In condition (8), the 
inequality holds for such points $(a, s)$ of the subdifferential $D^{-} u(t, x)$ which at the same time belong to the set $\partial u(t, x)$. In contrast to Definition 1 , the notion of viscosity solution [10] for equation (1) on the set $\bar{\Pi}_{T}$ requires that this solution satisfies inequality (8) at the boundary points $(t, x) \in \Gamma_{T}$ for all $(a, s) \in D^{-} u(t, x)$.

\subsection{On coercivity condition}

Conditions ensuring existence and uniqueness for viscosity solutions on the compact domain were obtained in [10]. One of the key conditions for the existence of viscosity solutions (see $[10,12])$ is the coercivity of the Hamiltonian:

$$
H(x, p) \rightarrow+\infty \quad \text { as } \quad|p| \rightarrow \infty
$$

It can be easily checked that the Hamiltonian (2) does not satisfy condition (22), for example, at $x=1$ and $x=-1$. Therefore, the existence theorems for viscosity solutions in the sense of paper [10] cannot be used in the problem (1)-(3).

Moreover, the notion of generalized viscosity solution is inapplicable to the initial value problem (1)-(3) on the compact set $\bar{\Pi}_{T}$ : If a viscosity solution would satisfy the condition $D^{-} u\left(t_{*}, x_{*}\right) \neq \varnothing$ at some point $\left(t_{*}, x_{*}\right) \in \Gamma_{T}$, then inequality (8) would not hold in this case. Indeed, let $0 \leq t_{*} \leq T$ and, for definiteness, $x_{*}=1$. Let $(a, s) \in D^{-} u\left(t_{*}, x_{*}\right)$. One can use the above definition of subdifferential to get the following inclusion:

$$
(a, s+k) \in D^{-} u\left(t_{*}, x_{*}\right) \quad \forall k \geq 0 .
$$

So, if condition (8) would hold then

$$
a+H(1, s+k)=a-f(x)+1-e^{2(s+k)} \geq 0, \quad \forall k \geq 0,
$$

which is obviously unfair. Therefore, we use the intersection of the subdifferential with the set $\partial u(t, x)$ (see condition (8)) in Definition 1 of a continuous generalized solution to the initial value problem (1)-(3) on the compact set $\bar{\Pi}_{T}$.

\subsection{Structure of subdifferentials on the border}

Let's consider the structure of the sets $D^{-} u(t, x)$ and $\partial u(t, x)$ for the function $u(\cdot)$ defined by (17)-(20) if $(t, x) \in \Gamma_{T}$.

In the case $0<t<T, \quad x=1$, we have

$D^{-} u(t, x)=D^{-} u(t, 1)=\{(f(1)-1, s) \mid s \in R, s \geq 0\}$,

$\partial u(t, x)=\partial u(t, 1)=\{(-H(1,-\infty),-\infty)\}=\{(f(1)-1,-\infty)\}$.

In the case $0<t<T, \quad x=-1$, we have

$D^{-} u(t, x)=D^{-} u(t,-1)=\{(f(-1)-1, s) \mid s \in R, s \leq 0\}$, $\partial u(t, x)=\partial u(t,-1)=\{(-H(-1, \infty), \infty)\}=\{(f(-1)-1, \infty)\}$.

Thus, for generalized solution (17)-(20)

$$
D^{-} u(t, x) \cap \partial u(t, x)=\varnothing, \quad(t, x) \in \Gamma_{T} .
$$


Acknowledgments. This work was partially supported by the Russian Foundation for Basic Research (project no. 14-01-00168) and the Complex Program of Ural Branch of RAS (project no. 15-16-1-11).

\section{References}

1. Saakian, D.B., Rozanova, O., Akmetzhanov, A.: Dynamics of the Eigen and the Crow-Kimura models for molecular evolution. Physical Review E - Statistical, Nonlinear, and Soft Matter Physics, vol. 78, no. 4, 041908 (2008)

2. Courant, R., Hilbert, D.: Methods of Mathematical Physics, vol. II. Interscience, New York (1962)

3. Subbotina, N.N., Shagalova, L.G.: On a solution to the Cauchy problem for the HamiltonJacobi equation with state constraints. Trudy Inst. Mat. i Mekh. UrO RAN. vol. 17, no.2, pp. 191-208 (2011)(in Russian)

4. Subbotina, N.N., Shagalova, L.G.: Construction of a Continuous Mini$\max /$ Viscosity Solution of the Hamilton JacobiBellman Equation with Nonextendable Characteristics. Trudy Inst. Mat. i Mekh. UrO RAN. vol. 20, no.4, pp. 247-257 (2014)(in Russian)

5. Subbotina, N.N., Shagalova, L.G.: On the continuous extension of a generalized solution of the Hamilton-Jacobi equation by characteristics that form a central field of extremals. Trudy Inst. Mat. i Mekh. UrO RAN. vol. 21, no.2, 220-235 (2015)(in Russian)

6. Shagalova, L.: Applications of Dynamic Programming to Generalized Solutions for Hamilton - Jacobi Equations with State Constraints. SOP Trans. on Applied Mathematics. vol. 1, no. 2, pp. 70-83 (2014)

7. Kruzhkov, S.N.: Generalized solutions of nonlinear equations of the first order with several variables. I. Matematicheskii Sbornik (N.S.), vol. 70(112), no. 3, pp. 394415 (1966) (in Russian)

8. Crandall, M.G., Lions, P.-L.: Viscosity solutions of Hamilton-Jacobi equations. Trans. Amer. Math. Soc., vol. 277., no 1, pp. 1-42 (1983)

9. Subbotin, A.I.: Generalized Solutions of First Order PDEs: The Dynamical Optimization Perspective. Birkhauser, Boston (1995)

10. Capuzzo-Dolcetta, I., Lions, P.-L.: Hamilton-Jacobi Equations with State Constraints. Trans. AMS, vol. 318, no. 2, pp. 643-683 (1990)

11. Clarke, F.: Optimization and nonsmooth analysis. John Wiley \& Sons, Inc., New York (1983)

12. Crandall, M.G., Newcomb, R.: Viscosity Solutions of Hamilton-Jacobi Equations at the Boundary. Proc. of the AMS, vol. 94, no. 2, pp. 283-290 (1985)

13. Rockafellar, R.T.: Convex Analysis. Princeton Univ. Press, Princeton, NJ (1970)

14. Pontryagin, L.S., Boltyanskii, V.G., Gamkrelidze, R.V., Mishchenko, E.F.: The Mathematical Theory of Optimal Processes. Pergamon Press, Oxford (1964)

15. Subbotina, N.N.: The method of characteristics for Hamilton-Jacobi equation and its applications in dynamical optimization. Modern Mathematics and its Applications. Vol. 20, pp. 2955-3091 (2004)

16. Subbotina, N.N., Kolpakova, E.A., Tokmantsev, T.B., Shagalova, L.G.: The method of characteristics for Hamilton-Jacobi-Bellman equation. RIO UrO RAN, Ekaterinburg (2013) (in Russian) 